\title{
A Secure and Scalable Rekeying Mechanism for Hierarchical Wireless Sensor Networks
}

\author{
Song GUO ${ }^{\dagger \mathrm{a})}$, An-Ni SHEN ${ }^{\dagger}$, Nonmembers, and Minyi GUO ${ }^{\dagger \dagger}$, Member
}

\begin{abstract}
SUMMARY Many applications of wireless sensor networks (WSNs) require secure group communications. The WSNs are normally operated in unattended, harsh, or hostile environment. The adversaries may easily compromise some sensor nodes and abuse their shared keys to inject false sensing reports or modify the reports sent by other nodes. Once a malicious node is detected, the group key should be renewed immediately for the network security. Some strategies have been proposed to develop the group rekeying protocol, but most of existing schemes are not suitable for sensor networks due to their high overhead and poor scalability. In this paper, we propose a new group rekeying protocol for hierarchical WSNs with renewable network devices. Compared with existing schemes, our rekeying method possesses the following features that are particularly beneficial to the resource-constrained large-scale WSNs: (1) robustness to the node capture attack, (2) reactive rekeying capability to malicious nodes, and (3) low communication and storage overhead.

key words: key management, group rekeying, hierarchical wireless sensor network, security
\end{abstract}

\section{Introduction}

Wireless sensor networks (WSNs) have been envisioned to be very useful for a broad spectrum of emerging civil and military applications [1]. However, sensor networks are also confronted with many security threats such as node compromise, routing disruption and false data injection, because they are normally operated in an unattended, harsh or hostile environment. Among all these threats, the WSNs are particularly vulnerable to the node compromise because sensor nodes are not tamper-proof devices. An adversary might easily capture the sensor devices to acquire their sensitive data and keys and then abuse them to further compromise the communication between other non-captured nodes. This typical threat is known as the node capture attack.

In order to conquer such problem, it is desirable to design key distribution protocols to support secure and robust pair-wise communication among any pair of sensors. To prevent from the node capture attack is a challenging task in sensor networks that have scarce resources in energy, computation and communication. Therefore, only lightweight energy efficient key distribution mechanisms are affordable.

\footnotetext{
Manuscript received August 3, 2009.

Manuscript revised October 8, 2009.

${ }^{\dagger}$ The authors are with the School of Computer Science and Engineering, The University of Aizu, Aizu-Wakamatsu-shi, 9658580 Japan.

${ }^{\dagger}$ The author is with the Department of Computer Science and Engineering, Shanghai Jiao Tong University, Shanghai 200240,
} China.

a)E-mail: sguo@u-aizu.ac.jp

DOI: 10.1587/transinf.E93.D.421
For example, the conventional asymmetric key cryptosystem, such as RSA [2] and Diffie-Hellman [3], can not be implemented in sensor nodes due to their very limited capacities.

WSNs can be broadly classified into flat WSNs and hierarchical WSNs. In a flat WSN, all senor nodes have the same computational and communication capacities. In a hierarchical WSN, however, some special sensor devices, called Cluster Head $(\mathrm{CH})$, have much higher capacities than other sensor nodes. By applying some clustering algorithms like [4], the whole set of sensor devices could be partitioned into several distinct clusters such that each cluster has at least one $\mathrm{CH}$. Under this arrangement, each sensor node forwards the generated packets to its local $\mathrm{CH}$ by short-range transmissions, and the $\mathrm{CH}$ then performs a pre-processing for the raw data received from all other senor nodes in the cluster and finally forwards the aggregated data to the sink node, or Base Station (BS), by long-range transmissions. It has been shown in [5] that a hierarchical architecture can provide better performance, in terms of communication overhead, than a flat architecture in such networks.

When a sensor network is deployed in unattended and hostile environments such as battlefield, the adversary may capture and reprogram some sensor nodes, or inject some malicious sensor nodes into the network and make the network accept them as legitimate nodes. After getting control of a few nodes, the adversary can mount various attacks from inside the network. For example, a compromised node (intruder) may inject false sensing reports or maliciously modify reports that go through it. Under such attacks, the sink may receive incorrect sensing data and make wrong decisions, which may be dangerous in scenarios such as battlefield surveillance and environmental monitoring.

To defend against such attack, the security mechanisms in WSNs are required. Most of existing key management schemes focus on the efficiency of bootstrapping session keys which has been intensively studied in the literature of WSNs [6]-[8]. The group key is to allow a group of sensor nodes to decrypt a broadcast message. In hierarchical WSNs, CHs broadcast some secrecy information to notify the sensor nodes in a group. Because the secrecy messages can not be disclosed to adversaries, the secure link between $\mathrm{CH}$ and sensor node is necessary. The communication cost will be decreased by broadcast using group key instead of by unicast using pair-wise keys. To control information access in a hierarchical sensor network, only authorized sensors should have the cryptographic keys by which they can 
decode the disseminated information. Thus, a group key management is required for such a hierarchical environment as it can implement different access control policies at each group and provide mechanisms for secure group communication by eliminating the compromised nodes.

Traditionally, once such key system is adopted, the whole security system is established and fixed. However, when the WSN runs for a long time using a fixed key, it enhances the probability for the adversaries to decrypt the key by analyzing the adequate messages eavesdropped or capturing some nodes. Under this circumstance, the entire network security might be threatened. Thus, it is necessary to update this key with a new key to maintain backward secrecy [9]. The idea is to prevent a node with the new key from going backwards in time to decipher previous content encrypted with prior keys. Likewise, when a node leaves, it is necessary to update the key to maintain forward secrecy [9]. The idea is to prevent a node from using an old key to continue to decrypt new content.

The rest of this paper is organized as follows. Section 2 gives an overview of related work. Section 3 presents our system model. Section 4 describes a group of protocols for our rekeying mechanism. Sections 5 and 6 evaluate the security and the performance of our proposal, respectively. Section 7 summarizes our findings.

\section{Related Work}

The group rekeying problem has been extensively studied in the context of secure multicast in WSNs. Zhang et al. proposed three improved schemes to deal with the cluster rekeying problem. In the B-PCGR scheme [10], each sensor node $S_{i}$ needs to pre-load a $t$-degree g-polynomial $g(x)$ in its storage. After deployment, $S_{i}$ randomly picks a bivariate $(t, \mu)$-degree e-polynomial $e_{s_{i}}(x, y)$ :

$$
e_{s_{i}}(x, y)=\sum_{i=0}^{t} \sum_{j=0}^{\mu} a_{i j} x^{i} y^{j}
$$

and constructs a new polynomial $g^{\prime}(x)$ as follows.

$$
g^{\prime}(x)=g(x)+e_{s_{i}}\left(x, s_{i}\right)
$$

For each member node $S_{j}(j \neq i)$ in the group, $S_{i}$ sends $e_{S_{i}}\left(x, S_{j}\right)$ to $S_{j}$ by unicast through a secure channel. After that, $S_{i}$ removes $g(x)$ and $e_{s_{i}}(x, y)$ immediately, but keeps the polynomial $g^{\prime}(x)$. In the rekeying phase, $S_{i}$ requests the secrecy polynomial shares, e.g., $e_{s_{i}}\left(c, S_{j}\right)$, from $\tau(\tau>\mu)$ member nodes, e.g., node $S_{j}$, to reconstruct the polynomial $e_{s_{i}}(c, y)$, in which $c$ is the current version of the group key. Having obtained $e_{s_{i}}(c, y), S_{i}$ can compute the new group key:

$$
g(c)=g^{\prime}(c)-e_{s_{i}}\left(c, s_{i}\right) .
$$

This scheme has a very high communication complexity because the secrecy polynomial needs to be exchanged between any pair of member nodes in the initial phase and each node need to collect the polynomial shares from at least $(\mu+1)$ member nodes to calculate group key in the rekeying phase.

Chadha et al. proposed a variant group key distribution scheme [11], in which the rekeying message from the group head can disallow the compromised nodes to renew their pair-wise key and group key. In the pre-loading phase, each sensor node $S_{i}$ is pre-loaded the secret values $h\left(S_{i}\right)$ and $\ell\left(S_{i}\right)$ obtained from a $2 t$-degree masking polynomial $h(x)$ and a $t$-degree concealing polynomial $\ell(x)$, respectively. This scheme assumes that each group head has the intrusion detection capacity. In the rekeying phase, the group head generates a $t$-degree secrecy polynomial $f(x)$ and constructs $w(x)$ as follows:

$$
w(x)=g(x) f(x)+h(x),
$$

where $g(x)$ is constructed using the Ids of all compromised nodes. Once $g(x)$ is evaluated at the Id of any malicious node, the result will be equal to 0 . The group head then broadcasts $w(x)$ and the Id list of all detected compromised nodes throughout the whole group members. Upon receiving the message, any non-revoked node $S_{i}$ can compute the new pair-wise key $f\left(S_{i}\right)$ between sensor node $S_{i}$ and the group head as follows:

$$
f\left(S_{i}\right)=\frac{w\left(S_{i}\right)-h\left(S_{i}\right)}{g\left(S_{i}\right)}
$$

To obtain the new group key, each sensor node $S_{i}$ must unicast $s\left(S_{i}\right)$, defined as

$$
s\left(S_{i}\right)=f\left(S_{i}\right)+\ell\left(S_{i}\right),
$$

to a set of its trust member nodes through secure channels. Once $t+1$ of such shares are received, senor node $S_{i}$ can derive the original polynomial $s(x)$ and use $s(0)$ as group keys in a new round of the rekeying phase. We notice that this polynomial reconstruction approach incurs high communication cost because each valid sensor node needs to broadcast the rekeying information to its group sensor member for the new group key calculation.

\section{System Model}

As in other hierarchical models of sensor network [12], [13], our system also assumes that a sensor network is divided into clusters, which are the minimum unit for detecting events. A cluster head coordinates all the actions inside a cluster and each pair of cluster heads in their transmission range can communicate directly with each other. Moreover, we assume a single base station (BS) or an access point (AP) in the network and works as the network controller to collect event data. As illustrated in Fig. 1, the information collected by cluster heads from all its sensor nodes is retrieved by a mobile AP periodically. During the information retrieval operation, the AP broadcasts a beacon to activate cluster heads in its coverage area. Activated cluster heads then transmit their data to the AP through a common wireless channel. 
Table 1 Comparison of group rekeying schemes with respect to several desired properties.

\begin{tabular}{|l|c|c|c|}
\hline & VGR(ours) & B-PCGR([10]) & Chadha's([11]) \\
\hline Fully collusion resistant against node capture attack & $\sqrt{ }$ & & \\
\hline Resistance to eavesdropping attack & $\sqrt{ }$ & $\sqrt{ }$ & $\sqrt{ }$ \\
\hline Rekeying not relying on member nodes & $\sqrt{ }$ & & \\
\hline Updating secrecy polynomials in every rekeying period & $\sqrt{ }$ & & $\sqrt{ }$ \\
\hline Backward secrecy & $\sqrt{ }$ & & \\
\hline Forward secrecy & $\sqrt{ }$ & & \\
\hline
\end{tabular}

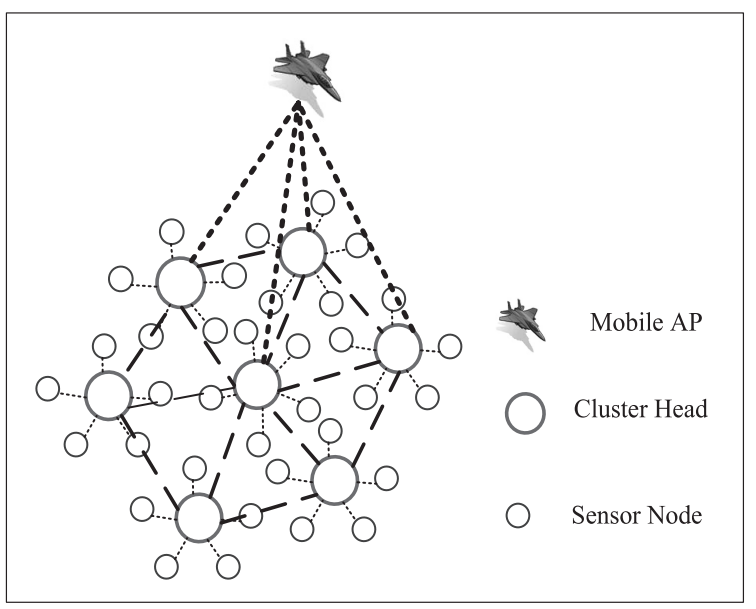

Fig. 1 A three-tier hierarchical WSN.

Our model has three different types of wireless sensor devices: base station $(\mathrm{BS})$, cluster head $(\mathrm{CH})$ and normal sensor node. The sensor network is administrated by an offline security server which is responsible for node initialization and deployment. Each low-cost sensor node has low data processing capability, limited memory storage and battery power supplies, and short radio transmission range. The CHs are equipped with richer resources (e.g., higher power batteries, large memory storages, powerful antenna, etc.) and higher data processing capacities, and thus can execute relatively complicated numerical operations. Each $\mathrm{CH}$ adopts the watchdog mechanism [14] as well that can monitor its group member in real-time and identify the compromised nodes when observing misbehaviors. As the most powerful node in a WSN, the BS has virtually unlimited memory storage capacity and sufficiently large radio transmission range to reach all other devices in a network.

In our network model, a large number of sensor nodes are arbitrarily deployed in a given area. After deployment, according to some existing clustering algorithms, e.g., [4], the network is partitioned into several distinct clusters. For each cluster, the cluster heads are placed at an appropriate location in the network.

\section{A Verification-Based Rekeying Scheme}

In general, the group rekeying in WSNs is challenging because of the potentially large network scale and the constrained system resources. The sensor nodes are often de- ployed in unattended and adversarial environments. Due to these challenges, a group rekeying protocol must meet the following requirements:

- Fully collusion resistant against captured attack - A group rekeying protocol should be able to fully defend against node captured attack because sensor nodes are low-cost devices and thus they cannot afford tamperresistant hardware. Recently, the physical attacks show that even memory chips with built-in tamper-resistance mechanisms are subject to various memory read-out attacks. Thus, the adversary may capture many sensor nodes and analyze them to obtain their related secrecy information. Our proposed protocol should support that the compromised node will not be revealing any key information of non-captured nodes.

- Resistance to eavesdropping attack - The adversary may collect and analyze the group rekeying messages to derive the new group key. Hence, such messages should not reveal any new group key information in our proposal protocol.

- Rekeying without relying on member nodes - Both BPCGR and Chadha's protocols distribute the polynomial shares to each member node and then delete the polynomial to increase the resilience of node captured attack. In the rekeying phase, these shares must be collected back for the reconstruction of the polynomial, which results in a high communication cost that is not efficient for WSNs. This motivates us to explore new approaches with low communication complexity.

- Periodical secrecy polynomial updates to achieve both backward and forward secrecy - If the $t$-degree polynomial for generating new group key does not change, all the group keys could be cracked once group keys are compromised by an adversary.

In summary, a practical group rekeying protocol for WSNs should be able to fully defend against node capture attack, to provide high resistance to eavesdropping attack, to generate new group keys in a scalable manner, and to achieve both backward and forward secrecy. Table 1 shows that neither of the overviewed group rekeying schemes can provide all these required properties except our proposed scheme.

In this section, we present a verification-based group rekeying (VGR) protocol based on a three-tier hierarchal network model. The proposed scheme should achieve the 
Table 2 Notations.

\begin{tabular}{|l|l|}
\hline Notation & Description \\
\hline$B S$ & A base station \\
\hline$C H_{a}$ & The Id of cluster head $a$ \\
\hline$S_{i}$ & The Id of sensor node $i$ \\
\hline$K_{a, b}$ & A pair-wise key between $a$ and $b$ \\
\hline$G K_{a}^{k}$ & A group key at version $i$ of group $a$ \\
\hline$E\left(d a t a, K_{a, b}\right)$ & A symmetric encryption function using key $K_{a, b}$ as key \\
\hline$g_{a}^{k}(x)$ & A bivariate polynomial function for group key regeneration at version $k$ of group $a$ \\
\hline$R_{i}^{k}$ & A random number generated by $S_{i}$ in the $k$-round group rekeying \\
\hline$n$ & The total number of sensor nodes \\
\hline$m$ & The total number of cluster heads \\
\hline$n_{c}$ & The average number of sensor nodes in a cluster \\
\hline$t, \mu$ & The degree of a polynomial \\
\hline$\omega$ & The number of compromised nodes in a cluster \\
\hline$\Omega_{a}$ & The set of all compromised nodes detected in cluster- $a$ \\
\hline$L_{K e y}$ & The number of bits of a key \\
\hline$L_{I d}$ & The number of bits of an Id \\
\hline
\end{tabular}

following goals.

1. The polynomial for group rekeying should be updated periodically to achieve both forward and backward secrecy.

2. Each sensor node doesn't need to preload extra information for supporting large-scale WSNs.

3. Once a compromised node is detected, a new group key should be generated immediately for the security of network.

In order to present VGR in a formal way, we list the notations used in our protocol descriptions in Table 2 for convenience to the reader.

\subsection{Key Predistribution}

We assume that there are $n$ sensor nodes and $m$ cluster heads in the network. Before all the nodes to be deployed into usage, some secrecy information should be pre-assigned into the three types of network devices as follows.

The BS has $(n+m)$ pair-wise keys to share with each sensor node and $\mathrm{CH}$, respectively.

For each sensor node $i$, the security server preloads it with:

- a unique Id denoted as $S_{i}$

- a pair-wise key $K_{B S, S_{i}}$ shared with BS with:

Similarly, each cluster head $a$ needs to be preloaded

- a unique Id denoted as $\mathrm{CH}_{a}$

- a pair-wise key $K_{B S, C H_{a}}$ shared with BS

After the key pre-assignment phase, wireless sensors are randomly distributed in a given area, and later on, some clustering algorithms, e.g., [4], shall organize the network into a hierarchical structure. We consider that each sensor node $i$ is assigned to one or more clusters, e.g., cluster $a$, after its deployment. Suppose that the pair-wise key between
$\mathrm{CH}_{a}$ and $\mathrm{S}_{i}$ can be established using some existing pair-wise key establishment technique.

\subsection{Group Key Establishment and Rekeying}

Let $\Omega_{a}$ a be the set of all compromised nodes detected in cluster- $a$. Now we consider the $k$-th $(k \geq 1)$ round of rekeying process described as follows.

- Step 1: Each sensor node $S_{i}$ generates a random number $R_{i}^{k}$ and sends $E\left(R_{i}^{k}, K_{C H_{a}, S_{i}}\right)$ to its cluster head $C H_{a}$ through the secure channel protected by their shared pair-wise key $K_{\mathrm{CH}_{a}, S_{i}}$. All these random numbers will be used to generate group keys by $\mathrm{CH}_{a}$.

- Step 2: $\mathrm{CH}_{a}$ generates a group key $G K_{a}^{k}$ and constructs the $k$-th version of group polynomial $g_{a}^{k}(x)$ using the random numbers recieved from all its member sensor nodes:

$$
\begin{aligned}
& g_{a}^{k}(x)=A_{a}^{k}(x)+G K_{a}^{k} \\
& A_{a}^{k}(x)=\prod_{S_{j} \notin \Omega_{a}}\left(x-R_{j}^{k}\right)
\end{aligned}
$$

where $\Omega_{a}$ is the set of all compromised nodes detected in cluster- $a$. In this way, for any sensor node $S_{i}$, the constructed polynomial has the following properties.

$$
\begin{aligned}
& A_{a}^{k}\left(R_{i}^{k}\right)=0, S_{i} \notin \Omega_{a} \\
& A_{a}^{k}\left(R_{i}^{k}\right) \neq 0, S_{i} \in \Omega_{a}
\end{aligned}
$$

- Step 3: $C H_{a}$ broadcasts the $k$-th version of group polynomial $g_{a}^{k}(x)$, each of whose coefficient is encrypted by the current group key $G K_{a}^{k-1}$, to all its member nodes.

- Step 4: After decrypting the received $k$-th version of group polynomial $g_{a}^{k}(x)$, the innocent sensor node $S_{i}$ $\left(S_{i} \notin \Omega_{a}\right.$ ) evaluates $g_{a}^{k}(x)$ at $x=R_{i}^{k}$ to obtain the new group key $G K_{a}^{k}$.

$$
g_{a}^{k}\left(R_{i}^{k}\right)=A_{a}^{k}\left(R_{i}^{k}\right)+G K_{a}^{k}=G K_{a}^{k}
$$




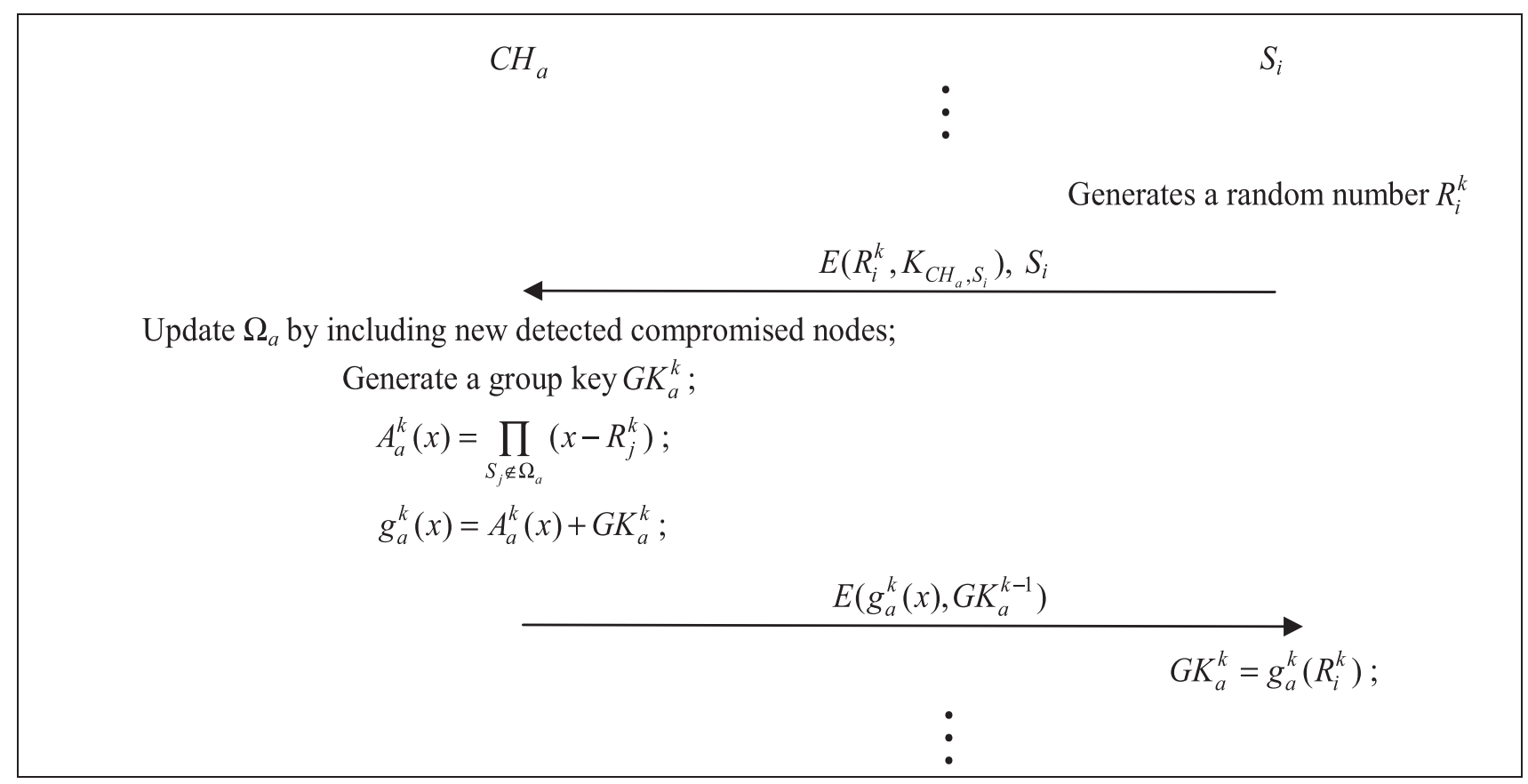

Fig. 2 The VGR protocol.

As illustrated in Fig. 2, the random number $R_{i}^{k}$ generated by $S_{i}$ is only known to $C H_{a}$ and $S_{i}$ because this random number is encrypted by their pair-wise key while being sent to the cluster head. For the security of network, the group key must be renewed once a malicious node is detected by $\mathrm{CH}_{a}$ or when the rekeying timer expires. For example, we assume a malicious/compromised node, e.g., $S_{i}$, is detected by $\mathrm{CH}_{a}$. The compromised node set $\Omega_{a}$ is then updated as $\Omega_{a}=\Omega_{a} \cup\left\{S_{i}\right\}$ and the corresponding factor $\left(x-R_{i}^{k}\right)$ is removed from the polynomial $A_{a}^{k}(x)$ as well, immediately. Upon receiving the broadcast message for rekeying, the compromised node $S_{i}$ cannot obtain the new group key by evaluating $g_{a}^{k}(x)$ at $x=R_{i}^{k}$, i.e., $G K_{a}^{k} \neq g_{a}^{k}\left(R_{i}^{k}\right)$, because $A_{a}^{k}\left(R_{i}^{k}\right) \neq 0$.

\section{Security Analysis}

We assume that the BS will never be compromised. After deployment, each cluster head and each sensor node can be captured and compromised by attackers due to the unattended deployment environments and their lack of tamperresistance. The adversary can read out all information stored in the node to get all secret information. Furthermore, the compromised nodes can be reprogrammed and thus fully controlled by the attackers.

In this paper, we consider the following variant types of attacks [15].

- Outsider attacks: launched by attackers that have not compromised any sensor nodes and therefore do not know any secret of network. In particular, the attackers may eavesdrop and collect messages, and attempt to derive some secrets from the message. This is denoted as type-I eavesdropping attack.
- Insider attacks: launched by attackers that have compromised some sensor nodes and therefore know some secrets preloaded to these compromised nodes. Certainly, insider attackers can also launch the above attacks denoted as type-II eavesdropping attack. In addition, the attackers may collect the secrets owned by compromised nodes, and attempt to derive the secrets held by innocent nodes (and therefore can cheat these innocent nodes or impersonate as them). This is the well-known node capture attack.

\subsection{Eavesdropping Attack}

Recall that in B-PCGR and Chadha's scheme, the message interactions for group key regeneration are all through secure unicast channels, which are guaranteed by the current pair-wise keys. This property can keep them from both typeI and type-II eavesdropping attacks. Let $L_{k e y}$ be the length of the group key. The probability to find out the new group key by eavesdropping is therefore $2^{-L_{k e y}}$.

Similarly, in our VGR protocol, the new group polynomial $g_{a}^{k}(x)$ is encrypted by the current group key $G K_{a}^{k-1}$ over the broadcast channel as shown in Fig. 2. Without knowing any secret information of the network, e.g. the current group keys, the attackers cannot derive the new group key by eavesdropping. In other words, our protocol is also resistant to type-I eavesdropping attacks. The results on the security analysis of type-II eavesdropping attacks to VGR are given in the following Theorem.

Theorem 1. If there are $\omega(\omega \geq 1)$ number of sensor nodes are already compromised in a cluster with $n_{c}$ nodes, the probability to derive the new group key of the VGR pro- 
Table 3 Security analysis.

\begin{tabular}{|l|c|c|c|}
\hline & VGR & B-PCGR & Chadha's \\
\hline Type-I eavesdropping attack & $2^{-L_{k e y}}$ & $2^{-L_{k e y}}$ & $2^{-L_{k e y}}$ \\
\hline Type-II eavesdropping attack & $\left(n_{c}-\omega+1\right) \cdot 2^{-L_{k e y}}$ & $2^{-L_{k e y}}$ & $2^{-L_{k e y}}$ \\
\hline Node capture attack & fully secure & $\min (\mu+1, t)$-security problem & $t$-security problem \\
\hline Backward Secrecy & support & not support & not support \\
\hline Forward Secrecy & support & not support & not support \\
\hline
\end{tabular}

tocol by eavesdropping is at most $\left(n_{c}-\omega+1\right) \cdot 2^{-L_{k e y}}$. Proof: Let $S_{i}$ be a compromised node that is detected just before the $k$-round of group rekeying procedure. The broadcast message $E\left(g_{a}^{k}(x), G K_{a}^{k-1}\right)$ can thus be overheard and decrypted successfully. If $S_{j}$ is an innocent node in the same cluster as $S_{i}$, its corresponding random number $R_{j}^{k}$ must not be disclosed. By randomly choosing a number $R$ with $L_{k e y}$ bits and substituting it to $g_{a}^{k}(R)$, the resulting value will be equal to the new group key if and only if $R=S_{j} \notin \Omega_{a}$ with $\left(n_{c}-\omega\right)$ different combinations. In addition, the attacker can use arbitrary checking for the new group key, resulting in the overall probability to derive the new group key to be at most $\left(n_{c}-\omega+1\right) \cdot 2^{-L_{k e y}} . \square$

\subsection{Node Capture Attack}

In this paper, we give a security analysis for our proposed rekeying scheme by comparing to other proposals in terms of robustness to the node capture attack.

Theorem 2. Our proposed VGR scheme can fully defend the node capture attack.

Proof: Based on the assumption of intrusion detection capability of each cluster head in the VGR scheme, any disclosed information $R_{i}^{k}$ (i.e., sensor node $S_{i}$ is compromised) will be removed from the new group polynomial $g_{a}^{k}(x)$ as shown in Sect. 4.2. Furthermore, the random numbers generated $R_{j}^{k}(j \neq i)$ at each uncompromised node $S_{j}$ is unknown to the adversary. In other words, the adversary can not cannot derive a value $c$ from the compromised nodes to evaluate $x=c$ in order to obtain the new group key, i.e., $g_{a}^{k}(c)=A_{a}^{k}(c)+G K_{a}^{k}=G K_{a}^{k}$. Once a sensor node is compromised, the disclosed information is just the current group key, which will expire shortly, and this information is no use for deriving any old or new group keys. Therefore, we can conclude that our CRGR scheme can fully defend the node capture attack.

On the other hand, the other two schemes suffer this attack as explained in the following theorems.

Theorem 3. The B-PCGR scheme has a $t$-security problem if each node capture happens in a different rekeying phase, or a $(\mu+1)$-security problem if all node captures happen in the same rekeying phase.

Proof: The first conclusion is obvious because the $t$-degree polynomial $g(x)$ is never updated in every rekeying period and it can be derived after $(t+1)$ previous group keys are compromised. Now, we consider sensor node $S_{i}$ itself and a total $(\mu+1)$ number of member nodes of $S_{i}$ are compromised by an adversary in the same rekeying phase. For each compromised member node $S_{j}$ of $S_{i}$, the secrecy polynomial $e_{S_{i}}\left(x, S_{j}\right)$ is then disclosed. After collecting $(\mu+1)$ of such polynomials, the adversary can derive the polynomial $e_{S_{i}}(x, y)$ because the degree of variable $y$ is $\mu$. Furthermore, the polynomial $g^{\prime}(x)$ is already disclosed from the compromised node $S_{i}$. Finally, the adversary can derive $g(x)=g^{\prime}(x)-e_{S_{i}}\left(x, S_{i}\right)$, which can used to calculate any expired or new group keys.

Theorem 4. The Chadha's scheme has a $2 t$-security problem if each node capture happens in an arbitrary rekeying phase, or a $t$-security problem if all node captures happen in the same rekeying phase.

Proof: Following a similar reasoning, we observe that if there are $(2 t+1)$ nodes are compromised in an arbitrary rekeying phase, both the $2 t$-degree polynomial $h(x)$ and the $t$-degree polynomial $\ell(x)$ can be derived. In any given rekeying phase, the corresponding polynomials $w(x)$ and $g(x)$ are public, resulting in that both $f(x)$ and $s(x)$ can be used to calculate the pair-wise key and group key, respectively, in this rekeying phase. Now, we consider total $(t+1)$ number of sensor nodes are compromised in the same rekeying phase. Both $t$-degree polynomials $\ell(x)$ and $s(x)$ can thus be derived. Therefore, the polynomial $h(x)$ can be derived as

$$
\begin{aligned}
& f(x)=s(x)-\ell(x), \\
& h(x)=w(x)-g(x) \cdot f(x) .
\end{aligned}
$$

In any given rekeying phase, let $w^{\prime}(x), g^{\prime}(x), f^{\prime}(x)$, and $s^{\prime}(x)$ be the polynomials corresponding to $w(x), g(x), f(x)$, and $s(x)$, respectively. The new polynomials for generating pairwise key and group key can be obtained as

$$
\begin{aligned}
& f^{\prime}(x)=\frac{w^{\prime}(x)-h(x)}{g^{\prime}(x)}, \\
& s^{\prime}(x)=f^{\prime}(x)+\ell(x) .
\end{aligned}
$$

Note that $w(x), g(x), w^{\prime}(x)$, and $g^{\prime}(x)$ in (12) - (15) are all public polynomials.

As we observe from the above theorems, after a number of sensor nodes are compromised, both B-PCGR and Chadha's schemes will disclose the polynomials that can 
Table 4 Performance analysis.

\begin{tabular}{|c|l|l|l|l|}
\hline & & B-PCGR & Chadha's & Our Scheme \\
\hline \multirow{3}{*}{ Computation } & Cluster head & N/A & $O(t)$ multiplications & $O\left(\left(n_{c}-\omega\right)^{2}\right)$ multiplications \\
\cline { 2 - 5 } & Sensor node & $\begin{array}{l}\tau \text { decryptions, } \\
O\left(\tau \cdot t^{2}+\mu^{3}\right) \text { mul./div. }\end{array}$ & $\begin{array}{l}t \text { decryptions, } \\
O\left(t^{3}\right) \text { mul./div. }\end{array}$ & $O\left(n_{c}-\omega\right)$ multiplications \\
\hline \multirow{2}{*}{ Communication } & Cluster head & N/A & $(2 t+1) \cdot L_{K e y}+\omega \cdot L_{I d}$ & $\left(n_{c}-\omega+1\right) \cdot L_{K e y}$ \\
\cline { 2 - 5 } & Sensor node & $\tau \cdot(\mu+2) \cdot L_{K e y}$ & $(t+1) \cdot L_{K e y}$ & $L_{K e y}$ \\
\hline \multirow{2}{*}{ Storage } & Cluster head & N/A & $(3 t+2) \cdot L_{K e y}$ & 0 \\
\cline { 2 - 5 } & Sensor node & $(t+1) \cdot L_{K e y}$ & $2 L_{K e y}$ & 0 \\
\hline
\end{tabular}

generate any group key in the past or future. On the contrary, our proposed protocol can achieve forward and backward secrecy because no such polynomials exist in our approach. The major results of security analysis in this section are summarized in Table 3.

\section{Performance Evaluation}

In this section, we evaluate the performance of our proposal by comparing with B-PCGR and Chadha's schemes. The performance metrics include the computational complexity, communication overhead, and storage overhead. Table 4 summarizes the performance results.

\subsection{Computational Complexity}

In the B-PCGR scheme, each sensor node $S_{i}$ first needs to evaluate the $(t, \mu)$-degree e-polynomials $e_{S_{i}}(x, y)$ at $y=S_{j}$ $(j \neq i)$ for at least $(\tau+1)$ different nodes, $e$.g., node $S_{j}$. This requires $O\left(\tau \cdot t^{2}\right)$ multiplications / divisions. After receiving $(\tau+1)$ shares $e_{S_{i}}\left(c, S_{j}\right)$, node $S_{i}$ also needs to solve a linear equation system:

$$
e_{S_{i}}\left(c, S_{j}\right)=\sum_{k=0}^{\mu} C_{k}\left(S_{j}\right)^{k}
$$

with $(\mu+1)$ variables $\left(C_{0}, C_{1}, \cdots, C_{\mu}\right)$, to finally construct the polynomial $e_{S_{i}}(c, y)$. This requires $O\left(\mu^{3}\right)$ multiplications / divisions. The overall computation complexity is therefore $O\left(\tau \cdot t^{2}+\mu^{3}\right)$ multiplications / divisions. We also note that the B-PCGR scheme is for pure flat WSNs, in which no CHs are deployed.

In the Chadha's group rekeying phase, each $\mathrm{CH}$ needs to evaluate the new group key, which needs $O(t)$ multiplications. After receiving $(t+1)$ polynomial shares from member nodes, each sensor node needs to decrypt them and to solve a $(t+1)$-variable linear equation system, which needs $O\left(t^{3}\right)$ multiplications / divisions.

In our group rekeying protocol, each $\mathrm{CH}$ constructs $g_{i}(x)$ using the updated $\left(n_{c}-\omega\right)$ random numbers, which needs $O\left(\left(n_{c}-\omega\right)^{2}\right)$ multiplications. After receiving the group rekeying request, each sensor node needs to evaluate the new group key, which needs $O\left(n_{c}-\omega\right)$ multiplications.

The above analysis shows that our scheme does not outperform other proposals significantly on the computational cost. However, in a typical WSN, the radio communications have much higher energy consumption than the code executions and calculations [6]. The experimental results in [16] show that the energy of sending 1 bit to $100 \mathrm{~m}$ away is roughly the same as exciting 3000 instructions in sensor nodes. The extremely low communication cost, as shown in the next section, allows our scheme to achieve minimal overall energy consumption compared with other proposals.

\subsection{Communication Overhead}

In the B-PCGR scheme, each sensor node $S_{i}$ sends out a $\mu$ degree polynomial $e_{S_{i}}\left(x, S_{j}\right)$ by unicast to each $S_{j}$ of a total $\tau(\tau>\mu)$ member nodes. All such unicast messages for each sensor node constitute $\tau \cdot(\mu+1) \cdot L_{K e y}$ bits. Subsequently, each sensor node $S_{i}$ collects $\tau$ secrecy polynomial shares $e_{S_{i}}\left(k, S_{j}\right)$ from its member nodes, e.g., $S_{j}$. Each of these shares has $L_{K e y}$ bits. The overall communication overhead of B-PCGR for each rekeying is therefore $\tau \cdot(\mu+2) \cdot L_{K e y}$ bits.

In the Chadha's scheme, each $\mathrm{CH}$ broadcasts a new $2 t$ degree polynomial $w(x)$ and $\omega$ Ids of detected compromised nodes to all the sensor nodes in the cluster. Such broadcast message has $(2 t+1) \cdot L_{K e y}+\omega \cdot L_{I d}$ bits. To derive the new group key at sensor node side, each sensor node $S_{i}$ must send out $t+1$ secrecy polynomial shares $s\left(S_{i}\right)$ to its member nods. All such unicast messages for each sensor node constitute $(t+1) \cdot L_{\text {Key }}$ bits.

In our proposed protocol, sensor nodes just transmit the new generated random number for rekeying and the only communication overhead at each cluster head is the broadcast message $g_{a}^{k}(x)$. It includes an encrypted $\left(n_{c}-\omega\right)$-degree polynomial with $\left(n_{c}-\omega+1\right) \cdot L_{K e y}$ bits.

As mentioned in Sect. 6.1, communication cost dominates the overall energy consumption. Using the results in Table 4, the normalized communication, i.e., the average bits transmitted at each node (both sensor node and cluster head) per rekeying phase, of our protocol can be obtained as:

$$
\begin{aligned}
\frac{m \cdot\left(n_{c}-\omega+1\right) \cdot L_{K e y}+n \cdot L_{K e y}}{m+n} \\
\quad=\frac{2 n-(\omega-1) \cdot m}{m+n} \cdot L_{\text {Key }} \\
\quad<2 L_{\text {Key }} .
\end{aligned}
$$

In other words, the average message complexity is $O(1)$. By a similar derivation, the Chadha's scheme has an average message complexity at order $O(t)$. In order to achieve comparable capacity of node compromise resistance as the 
VRG, the degree of the polynomial in Chadha's scheme should be at least equal to $n$, resulting in the complexity to be $O(n)$. Compared to this result, our proposed scheme is more scalable and can accomodate large-scale networks.

\subsection{Storage Overhead}

In the evaluation of storage overhead, we consider the space requirement of the preloaded information in each sensor node and cluster head for the rekeying schemes.

In the B-PCGR scheme, each sensor node is pre-loaded a $t$-degree polynomial $g(x)$, which takes $(t+1) \cdot L_{K e y}$ bits in its storage for $(t+1)$ coefficients.

In Chadha's scheme, each $\mathrm{CH}$ is pro-loaded a $2 t$-degree polynomial function $h(x)$ and a $t$-degree polynomial function $\ell(x)$. All coefficients for the two polynomials require $(3 t+2) \cdot L_{K e y}$ bits. Each sensor node $S_{i}$ needs to store two secret values $h\left(S_{i}\right)$ and $\ell\left(S_{i}\right)$ with $2 L_{K e y}$ bits.

Except the preloaded information for secure pair-wise communications, our proposed scheme does not need to preload any information for group key establishment and group rekeying.

\section{Conclusion}

In this paper, we present a verification-based group rekeying scheme based on a three-tier WSN. Our scheme can fully defend against node capture attack and deny the malicious nodes to join group rekeying process. Our proposed scheme also achieves both forward and backward secrecy. Compared with the B-PCGR and Chadha's scheme, our scheme is more efficient and scalable in terms of computational complexity, communication overhead, and storage overhead. These features are particularly beneficial to support large-scale and resource constrained WSNs.

\section{Acknowledgements}

The authors wish to thank the anonymous referees for several constructive suggestions that have improved the quality of this paper. This work is supported by the JSPS (Japan Society for the Promotion of Science) Grant-in-Aid for Scientific Research and the National Natural Science Foundation of China under Grant Nos. 60811130528, 60725208, and 60533040 .

\section{References}

[1] I.F. Akyildiz, W. Su, Y. Sankarasubramaniam, and E. Cayirci, "Wireless sensor networks: A survey," J. Computer Networks, vol.38, no.4, pp.393-422, March 2002.

[2] R. Rivest, A. Shamir, and L. Adleman, "A method for obtaining digital signatures and public key cryptosystems," Commun. ACM, vol.21, no.2, pp.120-126, 1978.

[3] W. Diffie and M.E. Hellman, "New direction in cryptography," IEEE Trans. Inf. Theory, vol.22, no.6, pp.644-654, Nov. 1976.

[4] W.R. Heinzelman, A.P. Chandrakasan, and H. Balakrishnan, "An application specific protocol architecture for wireless microsensor networks," IEEE Trans. Wireless Commun., vol.1, no.4, pp.660-670,
Oct. 2002.

[5] Y. Cheng and D.P. Agrawal, "A improved key distribution mechanism for large-scale hierarchical wireless sensor networks," J. Ad Hoc Networks, vol.5, no.1, pp.35-48, Jan. 2007.

[6] Y. Cheng and D.P. Agrawal, "Efficient pairwise key establishment and management in static wireless sensor networks," IEEE Conference on Mobile Ad-hoc and Sensor Systems, Nov. 2005.

[7] W.L. Du, J. Deng, Y. Han, and P.K. Varshney, "A pairwise key predistribution scheme for wireless sensor network," ACM Conference on Computer and Communications Security, pp.42-51, Oct. 2003.

[8] L. Eschenauer and V. Gligor, "A key-management scheme for distributed sensor networks," ACM Conference on Computer and Communications Security, pp.41-47, Nov. 2002.

[9] S. Mishra, "Key management in large group multicast," Technical Report, CU-CS-970-02, Univ. of Colorado, 2002.

[10] W. Zhang and G. Cao, "Group rekeying for filtering false data in sensor networks: A predistribution and local collaboration-based approach,” IEEE INFOCOM, pp.503-514, 2005.

[11] A. Chadha, Y. Liu, and S.K. Das, "Group key distribution via local collaboration in wireless sensor networks," IEEE SECON 2005, pp.46-54, 2005.

[12] G. Jolly, M.C. Kuscs, P. Kokate, and M. Younis, "A lowenergy management protocol for wireless sensor networks," IEEE International Symposium Computers and Communication, pp.335-340, 2003.

[13] W. Zhang, H. Song, S. Zhu, and G. Cao, "Least privilege and privilege deprivation: Towards to tolerating mobile sink compromises in wireless sensor networks," ACM MobiHoc, pp.378-389, 2005.

[14] S. Marti, T. Giuli, and K. Lai, "Mitigating routing misbehavior in mobile ad hoc networks," ACM MobiCom, pp.255-265, 2000.

[15] W. Zhang, N. Subramanian, and G. Wang, "Lightweight and compromise-resilient message authentication in sensor networks," IEEE INFOCOM, pp.1418-1426, April 2008.

[16] L.M. Sun, J.Z. Li, Y. Chen, and H.S. Zhu, Wireless Sensor Networks, Tsinghua University Press, 2005.

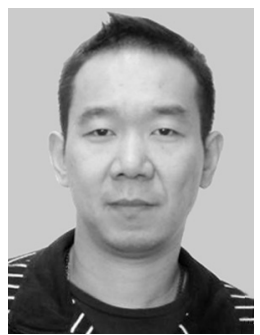

Song Guo received the Ph.D. degree in Computer Science from the University of Ottawa, Canada in 2005. He then held a position with the University of British Columbia on an NSERC (Natural Sciences and Engineering Research Council of Canada) postdoctoral fellowship. From 2006 to 2007, he was an Assistant Professor at the University of Northern British Columbia, Canada. He is currently an Assistant Professor at School of Computer Science and Engineering, the University of Aizu, Japan. His research interests are mainly in the areas of protocol design and performance analysis for computer and telecommunication networks, presently focusing on modeling, analysis, cross-layer optimization, and performance evaluation of wireless ad hoc and sensor networks for reliable, energyefficient, and cost effective communications. 


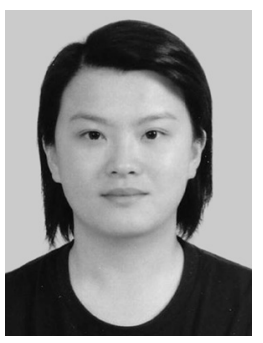

An-Ni Shen received her B.S. degree and M.S. degree both in Information Management from South Taiwan University, Taiwan, in 2006, and from Chaoyang University of Technology, Taiwan, in 2008, respectively. Since 2008, she has been a Ph.D. student in the Department of Computer Science and Engineering at the University of Aizu, Japan. Her main research interests are in security for wireless sensor networks.

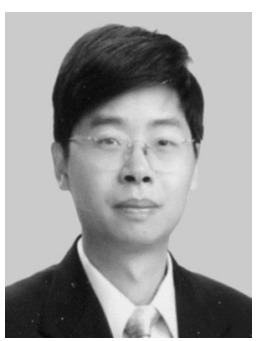

Minyi Guo received his Ph.D. degree in Computer Science from the University of Tsukuba, Japan. Before 2000, Dr. Guo had been a research scientist of NEC Corp., Japan. He is now a distinguished chair professor at the Department of Computer Science and Engineering, Shanghai Jiao Tong University, China, and a professor of the School of Information Engineering, Dalian Maritime University. He was also a visiting professor of Georgia State University, USA, Hong Kong Polytechnic University, University of Hong Kong, National Sun Yet-Sen University in Taiwan, University of Waterloo, Canada and University of New South Wales, Australia. He was also a professor of the University of Aizu, Japan. Dr. Guo has published more than 150 research papers in international journals and conferences. Dr. Guo has served as general chair, program committee, or organizing committee chair for many international conferences. He is the founder of International Conference on Parallel and Distributed Processing and Applications (ISPA), and International Conference on Embedded and Ubiquitous Computing (EUC). He is the editor-in-chief of the Journal of Embedded Systems. He is also on the editorial board of Journal of Pervasive Computing and Communications, International Journal of High Performance Computing and Networking, Journal of Embedded Computing, Journal of Parallel and Distributed Scientific and Engineering Computing, and International Journal of Computer and Applications. Dr. Guo's research interests include parallel and distributed processing, parallelizing compilers, pervasive computing, embedded software optimization, molecular computing, and software engineering. He is a senior member of IEEE, a member of the ACM, and IPSJ. 